\title{
miR-22 and miR-23a Control Glycerol-Dependent Gluconeogenesis by Regulating Aquaporin 9 Expression
}

Dwi Setyowati Karolina', Arunmozhiarasi Armugam¹, Sugunavathi Sepramaniam¹, Sharon Li Ting Pek², Michael TK Wong², Su Chi Lim², Chee Fung Sum ${ }^{2}$, Subramaniam Tavintharan ${ }^{2}$, and Kandiah Jeyaseelan ${ }^{1,3 *}$

${ }^{1}$ Department of Biochemistry, Yong Loo Lin School of Medicine, National University Health System,14 Medical Drive, Singapore

${ }^{2}$ Diabetes Centre, Khoo Teck Puat Hospital, Singapore

${ }^{3}$ Department of Anatomy and Developmental Biology, School of Biomedical Sciences, Faculty of Medicine, Nursing and Health Sciences, Monash University, Clayton, Victoria, Australia

\begin{abstract}
In obese individuals, a high efflux of glycerol from accumulated fat in adipose tissue into the liver is known to be associated with the development of type 2 diabetes. Aquaporin 9 (AQP9) is an aquaglyceroporin which serves as the primary route of hepatic glycerol uptake for gluconeogenesis. Hence, development of AQP9 blockers/regulators may be of potential benefit in controlling hyperglycaemia especially in obese and diabetic individuals. HTS13286 was recently identified as a specific $A Q P 9$ inhibitor, even so its limited solubility renders the molecule unsuitable for in vivo application. microRNAs are naturally occurring gene regulators that are often associated with various diseases. The feasibility in selective modulation of microRNAs has introduced a new paradigm for therapeutic applications.

In this study, we explore the possibility of using microRNAs to regulate AQP9 expression and eventually glycerolbased gluconeogenesis. In silico prediction of microRNAs targeting the 3' untranslated region of AQP9 was conducted using miRWalk database. Among the list of potential microRNAs, miR-22 and miR-23a were shortlisted for their high expression in liver and further confirmed to interact with $A Q P 9$ via luciferase assay. Over-expression of miR-22 or miR23a was able to reduce $A Q P 9$ expression (mRNA and protein) and ultimately inhibit glycerol-based gluconeogenesis in HepG2 cells. Livers of diabetic rats were also observed to exhibit an inverse correlation between miR-22, miR-23a and $A Q P 9$ expression.
\end{abstract}

As negative modulators of $A Q P 9$ expression, miR-22 and miR-23a suggest a potential role in regulating glycerol entry into hepatocytes which could be beneficial in managing glycerol-dependent hyperglycaemic conditions.

Keywords: microRNAs; Gene regulation; Aquaporin 9; Glycerol uptake; Glycerol gluconeogenesis

\section{Introduction}

Glycerol-based gluconeogenesis (glycerogluconeogenesis) is observed to be a contributing factor in increased endogenous glucose output in obese Type 2 diabetes (T2D) [1]. This is possibly due to expanded insulin-resistant adipose tissue mass and accelerated lipolysis that consequently increase glycerol availability for gluconeogenesis [1]. Hepatic glycerol uptake is mediated primarily by $A Q P 9$, an aquaglyceroporin family member which is found to be regulated by insulin [2,3]. Lepr ${ }^{d b} /$ Lepr $^{d b} A Q P 9^{-1-}$ mice were found to exhibit lower postprandial plasma glucose levels compared with those of $L e p r^{d b} / L e p r^{d b}$ $A Q P 9^{+/-}$control mice [4]. These results suggested that the absence of $A Q P 9$ as an entrance port for glycerol reduced the capacity of hepatic glycerogluconeogenesis which could be useful in diabetes treatment. A chemical compound, HTS13286 has recently been identified as an efficient inhibitor of $A Q P 9$. Nevertheless due to the limited solubility of HTS13286, this compound is currently considered unsuitable for in vivo application [5].

microRNAs (miRNAs) are gene regulators that can be dysregulated in diseases including T2D. The extensive implication of miRNAs across several pathophysiological conditions illustrates the potential of developing miRNA-based therapeutics. The feasibility in manipulating miRNAs expression makes them attractive candidates as therapeutic agents/targets. Throughout the years, several efforts have been made to optimize efficacy, stability and specificity of miRNA therapeutics for in vivo applications [6,7]. Although most miRNA therapeutics is still in preclinical development, two of them have progressed into clinical trials showing promising results: miravirsen (NCT01200420) and MRX34 (NCT01829971). A few miRNAs have been implicated in the regulation of gluconeogenesis [8-10]. Yet, none was associated with $A Q P 9$ expression and glycerol transport. In view of this and the fact that $A Q P 9$ inhibitor could be of therapeutic benefit in controlling gluconeogenesis in glycerol-based hyperglycaemic conditions, the potential of using endogenously expressed miRNAs to regulate AQP9 expression is explored in this study.

\section{Materials and Methods}

\section{Transfection of miRNAs in HepG2 cells}

HepG2 were purchased from American Type Culture Collection (ATCC) and grown in $5 \% \mathrm{CO}_{2}$ at $37^{\circ} \mathrm{C}$ in DMEM containing $5.5 \mathrm{mM}$ glucose supplemented with $10 \%$ FBS, $100 \mathrm{U}$ penicillin/ml, $100 \mu \mathrm{g}$ streptomycin $/ \mathrm{ml}$. Transfection of miRNAs was carried out according to Karolina et al. [11]. Anti- or pre-miR-22 and miR-23a were purchased from Ambion, USA. Briefly, respective miRNAs were diluted in $50 \mu \mathrm{l}$

*Corresponding author: Kandiah Jeyaseelan, Department of Biochemistry, Yong Loo Lin School of Medicine, National University Health System, 14 Medical Drive, Singapore 117599, Tel: +65-6516 3248; Fax: +65-6779 1453; E-mail: bchjeya@nus.edu.sg

Received April 18, 2014; Accepted May 28, 2014; Published June 05, 2014

Citation: Karolina DS, Armugam A, Sepramaniam S, Pek SLT, Wong MTK, et al. (2014) miR-22 and miR-23a Control Glycerol-Dependent Gluconeogenesis by Regulating Aquaporin 9 Expression. Metabolomics S2: 002. doi:10.4172/21530769.S2-002

Copyright: (c) 2014 Karolina DS, et al. This is an open-access article distributed under the terms of the Creative Commons Attribution License, which permits unrestricted use, distribution, and reproduction in any medium, provided the original author and source are credited. 


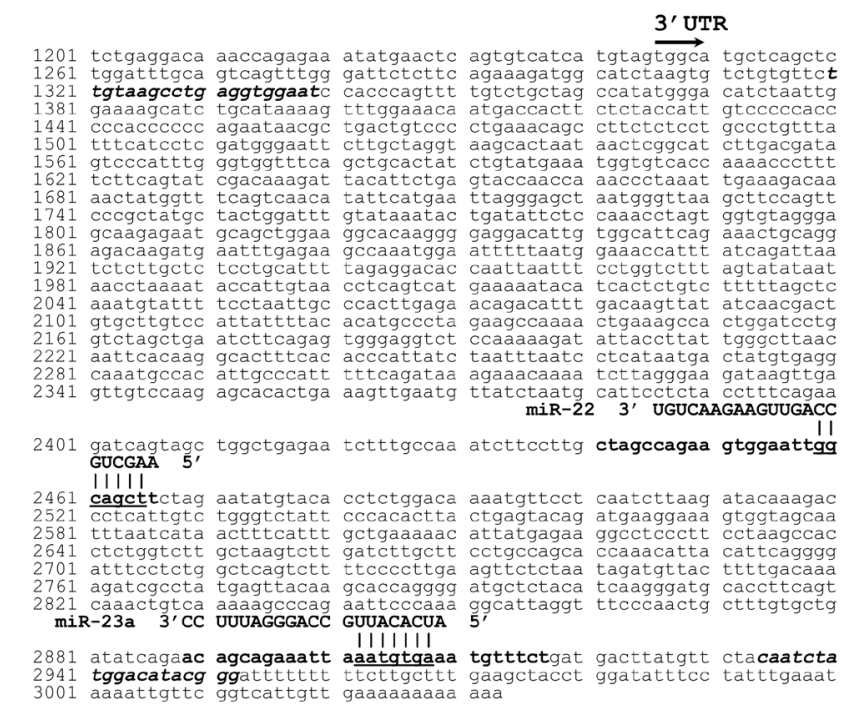

Figure 1: 3'UTR of $A Q P 9$ mRNA. The 3'UTR sequence of $A Q P 9$ mRNA was retrieved from NCBI (NM_020980.3). The seed region of miR-22 and miR23a is underlined. Forward 5'-TTGTAAGCCTGAGGTGGAAT-3' and Reverse 5'-CCCGTATGTCCATAGATTG-3' primers used for amplification of the 3'UTR are shown in italics bold fonts.

of Opti-MEM (Ambion, USA) to a final concentration of $30 \mathrm{nM}$. The mix was then complexed with $1 \mu \mathrm{l}$ of NeoFX in $50 \mu \mathrm{l}$ of Opti-MEM. HepG2 cells were transfected with these complexes and maintained for 48 hours prior to subsequent RNA/protein extraction. Total RNA (+miRNAs) was extracted from cells by a single-step method using TRIzol (Invitrogen Life Technologies, USA) according to the manufacturer's protocol.

\section{Real-time quantitative PCR}

Reverse transcription followed by real time quantitative PCR (qPCR) were carried out according to Karolina et al [11]. Specific primer sequences for $A Q P 9$ were generated using Primer Express (Hsa Forward 5'-GGCTGTGTTGCCCAAGCTA-3' and Reverse 5'-TGAAAATCCAACATTGATAGTGATGAC and Rno Forward 5'-GAAAATGCAACAGCATTCAT-3' and Reverse 5'GGCACCTGGCGTGGATAT). Specific primer probes for miR-22 (Assay ID: 000398) and miR-23a (Assay ID: 000399) were purchased from Applied Biosystems, USA. Real time quantitative PCRs were performed according to the manufacturer's protocols on Applied Biosystems 7900 HT sequence detection system. Data was normalized against the housekeeping gene 18S RNA.

\section{Luciferase and glucose production assays}

The 3'UTR of AQP9 mRNA was cloned into the Firefly luciferase expressing vector (pMIR-REPORT ${ }^{\mathrm{TM}}$; Ambion, USA) at the SpeI and HindIII sites (Figure 1). Plasmid and miRNA transfection procedure was adapted from Karolina et al. [11]. The cells were lysed $48 \mathrm{~h}$ post transfection and dual luciferase assay (Promega, USA) was carried out to quantitate the effects of anti- or pre-miR-22 or miR-23a interaction with the 3'UTR of $A Q P 9$ according to the manufacturer's protocol. For glucose assay, HepG2 cells were seeded at a density of $7 \times 10^{4}$ per well in 24-well plates and miRNA transfection was carried out immediately. After 48 hours, the medium was replaced with glucose and phenol red free DMEM (Sigma, USA) in which main gluconeogenic precursors such as lactate, glutamine and pyruvate are absent $[12,13]$. Cells were supplemented with $0.5 \mathrm{mM}$ of glycerol and incubated for 8 hours to allow for glucose production to take place $[5,14]$. After which, medium was collected for glucose assay (Sigma, USA) according to the manufacturer's protocol. Untreated cells that were not supplemented with glycerol were used as a blank. Cells subjected to the same transfection protocol using scrambled miRNAs were used as controls.

\section{Western blot analysis}

Western blot was carried out as described in Karolina et al. [11] The membranes were probed with primary rabbit anti-AQP9 antibodies (33 k Da, Santa Cruz Biotechnology, USA), at a concentration of $1 \mu \mathrm{g} /$ $\mathrm{ml}$ dilution in $0.5 \%$ blocking solution for 60 minutes. $\beta$-actin was used as a loading control (Bio-Rad, USA). Secondary antibodies (HRPconjugated goat anti-rabbit; Bio-Rad, USA) were used at 1:5000 dilution in $0.5 \%$ blocking solution. Following that, membranes were visualized with Super-Signal West-Dura Extended Duration Substrate (Pierce, Biotechnology, USA) and developed in Kodak Biomax film. Films of Western blots were scanned (Acer SWZ3300U), and the labeling intensities of the bands were quantified using ImageJ software (National Institutes of Health). Relative expression of protein is the quantity of band intensity expressed as a proportion to that in control samples.

\section{Rat model of T2D}

Male Wistar rats, 6 weeks of age and weighing approximately 150 g were used in this study. The animals were handled according to the guidelines of the Council for International Organization of Medical Sciences on Animal Experimentation (World Health Organization, Geneva, Switzerland) and the National University of Singapore. The animal protocols were approved (Protocol number: 062/09) by the National University of Singapore Institutional Animal Care and Use committee (NUS IACUC). Induction of Type 2 diabetes was done as described in Karolina et al. [11]. Diabetic condition was confirmed by oral glucose tolerance test [11] and animals that exhibited a state of hyperglycemia (fasting glucose $\geq 25 \mathrm{mmol} / \mathrm{L}$ ) and insulin resistance were selected for the study. At the end of the experimental period, animals were euthanized using $\mathrm{CO}_{2}$ and the liver tissue was harvested for gene expression studies.

\section{Statistical analysis}

Data were analyzed for statistical differences by performing twotailed t-tests between "control" and "test" sample groups with a level of significance set at $P<0.05$. All data are reported as mean \pm SEM.

\section{Results}

\section{miR-22 and miR-23a directly target $A Q P 9$}

Potential miRNA regulators of $A Q P 9$ were collated from the miRWalk prediction database (www.ma.uni-heidelberg.de/apps/zmf / mirwalk/). A total of 25 out of 492 miRNAs was shortlisted based on the highest number of predictions and their expression level in the human liver tissue [15] and HepG2 cells [16] are shown in Table 1. miR-22, miR-23a and miR-23b which showed the highest expression were selected. Also, since miR-23a and miR-23b share the same seed sequence and that they only differ by one nucleotide at the 3 ' end of the sequence, miR-23a was selected for subsequent interaction studies alongside miR-22. Figure 1 shows the predicted binding site of miR-22 and miR-23a at the 3'UTR of AQP9 mRNA. When the AQP9 3'UTR construct was co-transfected with either anti- miR-22 or miR-23a, an approximate $60-70 \%$ increase in luminescence was noticed (Figure 2). In contrast, independent transfection of pre- miR-22 or miR-23a 
Citation: Karolina DS, Armugam A, Sepramaniam S, Pek SLT, Wong MTK, et al. (2014) miR-22 and miR-23a Control Glycerol-Dependent Gluconeogenesis by Regulating Aquaporin 9 Expression. Metabolomics S2: 002. doi:10.4172/2153-0769.S2-002

Page 3 of 5

\begin{tabular}{|c|c|c|c|c|c|c|c|}
\hline Hsa-MicroRNA & miRanda & miRDB & miRWalk & RNA22 & Targetscan & $\begin{array}{l}\text { Average Signal Intensity in } \\
\text { HepG2 cells [16] }\end{array}$ & $\begin{array}{l}\text { Average Signal Intensity in Human Liver } \\
\text { Sample [15] }\end{array}$ \\
\hline miR-18a & 1 & 0 & 1 & 1 & 1 & 6.939 & 8.10 \\
\hline miR-18b & 1 & 0 & 1 & 1 & 1 & 5.305 & 2.86 \\
\hline miR-146a & 1 & 1 & 1 & 0 & 1 & 0.052 & 8.70 \\
\hline miR-146b-5p & 1 & 1 & 1 & 0 & 1 & 0.052 & 7.40 \\
\hline miR-181a & 1 & 0 & 1 & 1 & 1 & 3.751 & 10.45 \\
\hline miR-181b & 1 & 0 & 1 & 1 & 1 & 4.592 & 8.94 \\
\hline miR-181c & 1 & 0 & 1 & 1 & 1 & 2.203 & 2.27 \\
\hline miR-181d & 1 & 0 & 1 & 1 & 1 & 2.485 & 2.62 \\
\hline miR-198 & 1 & 1 & 1 & 1 & 1 & 1.286 & 2.06 \\
\hline miR-22 & 1 & 0 & 1 & 1 & 1 & 8.602 & 12.55 \\
\hline miR-23a & 1 & 1 & 1 & 0 & 1 & 10.945 & 11.98 \\
\hline$m i R-23 b$ & 1 & 1 & 1 & 0 & 1 & 8.885 & 13.47 \\
\hline miR-205 & 1 & 0 & 1 & 1 & 1 & 0.052 & 1.60 \\
\hline miR-324-5p & 1 & 1 & 1 & 1 & 1 & 6.078 & 7.17 \\
\hline miR-330-3p & 1 & 1 & 1 & 0 & 1 & 2.820 & 3.23 \\
\hline miR-331-3p & 1 & 1 & 1 & 0 & 1 & 6.541 & 5.27 \\
\hline miR-380 & 1 & 1 & 1 & 0 & 1 & 0.052 & 1.86 \\
\hline miR-488 & 1 & 0 & 1 & 1 & 1 & 0.052 & 1.94 \\
\hline miR-515-3p & 1 & 0 & 1 & 1 & 1 & 0.052 & 1.57 \\
\hline miR-522 & 1 & 1 & 1 & 0 & 1 & 0.052 & 1.68 \\
\hline miR-545 & 1 & 1 & 1 & 0 & 1 & 2.773 & 1.63 \\
\hline miR-590-3p & 1 & 1 & 1 & 0 & 1 & 0.052 & 1.72 \\
\hline miR-610 & 1 & 1 & 1 & 0 & 1 & 0.052 & 1.64 \\
\hline miR-648 & 1 & 1 & 1 & 0 & 1 & 0.052 & 2.39 \\
\hline miR-802 & 1 & 1 & 1 & 0 & 1 & 0.052 & 1.67 \\
\hline
\end{tabular}

Table 1: miRNAs predicted to target the 3'UTR of AQP9. A total of 25 miRNAs with the highest number of database predictions is shortlisted. Expression level of the 25 miRNAs in human liver sample as well as in HepG2 cells is shown. Among these, miR-22, miR-23a and miR-23b (in bold italics) were found to be highly expressed in both liver tissue and HepG2 cells.

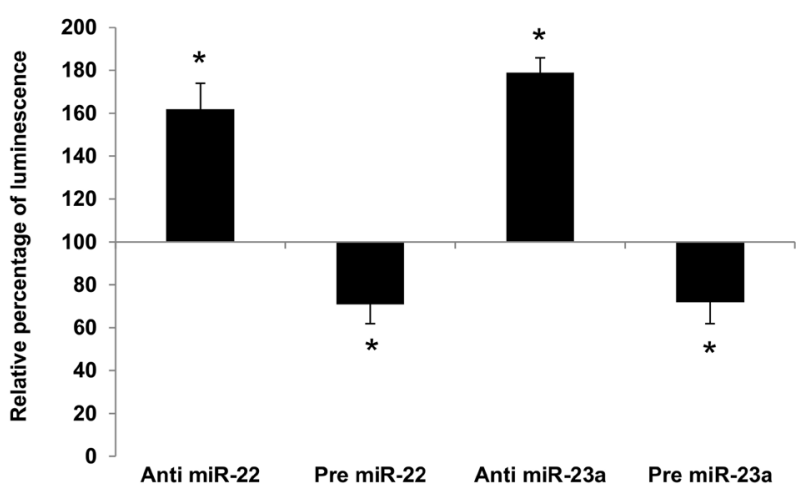

Figure 2: Dual luciferase reporter assay quantitation of the effects of anti- or pre- miR-22 or miR-23a interaction with the 3' UTR of AQP9. The plasmid constructs together with anti- or pre- miR-22 or miR-23a (30 nM) were co-transfected independently into HepG2 cells. Relative luminescence for luciferase gene activity in treated samples were obtained 48 hours posttransfection by normalizing the values against control transfected with corresponding anti or pre scrambled miRNAs. Data are presented as mean \pm SEM $(n=3)$ against control. Statistically significant differences are tested at ${ }^{*} p<0.05$ significance. resulted in a significant suppression of the reporter gene expression $(\sim 30 \%)$. Next, we elucidate the effects of modulating the miRNA levels on AQP9 expression. The presence of either anti- miR-22 or miR-23a increased $A Q P 9$ expression at both mRNA and protein levels significantly (Figure 3 ). Conversely, over-expression of either miRNA using their respective precursors was able to bring about reduction in $A Q P 9$ mRNA which was also reflected in the protein (Figure 3). However, the decrease in $A Q P 9$ protein in pre miR-23a treated cells did not reach a significant level (p-value: 0.06 ). In addition, $A Q P 9$ mRNA and protein were also found to be increased in the livers of diabetic rats while both miR-22 and miR-23a exhibited significant down-regulation (Figure 4). Altogether, these observations confirmed miR-22 and miR$23 \mathrm{a}$ as negative modulators of $A Q P 9$ expression.

\section{Regulatory roles of miR-22 and miR-23a on glycerogluconeogenesis}

Having confirmed that miR-22 and miR-23a can control AQP9 expression which primarily regulates glycerol transport in hepatocytes [5], we subsequently investigated whether glycerogluconeogenesis could be controlled by modulating miR-22 and miR-23a expression. To perform this, HepG2 cells were transfected with corresponding anti- or pre-miR-22 or miR-23a prior to glucose assay. Forty eight hours (48 
Citation: Karolina DS, Armugam A, Sepramaniam S, Pek SLT, Wong MTK, et al. (2014) miR-22 and miR-23a Control Glycerol-Dependent Gluconeogenesis by Regulating Aquaporin 9 Expression. Metabolomics S2: 002. doi:10.4172/2153-0769.S2-002

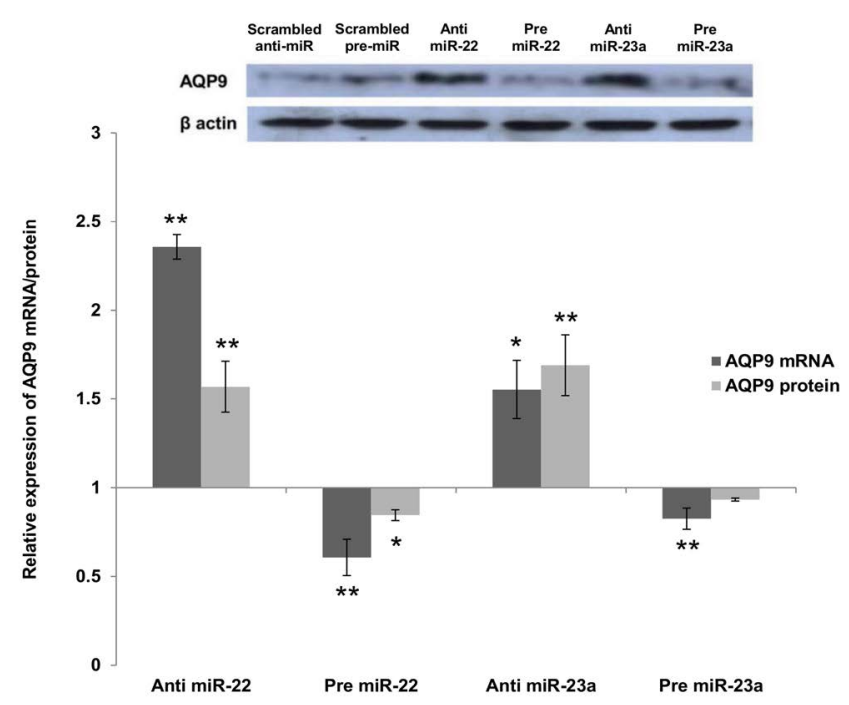

Figure 3: Relative expression of miR-22, miR-23a and $A Q P 9$ in HepG2 cells transfected with either anti- or pre- miR-22 or miR-23a (30 nM). Data was normalized against the housekeeping gene 18S RNA and presented as mean \pm SEM $(n=3)$ against control cells transfected with corresponding ant or pre scrambled miRNAs. Western Blot analysis of $A Q P 9$ protein in treated HepG2 cells is also shown (see insert). $\beta$-actin was used as loading control. The data presented here is a representative of three independent experiments. Statistically significant differences are tested at $p<0.05$ significance. ${ }^{*} p<0.05$, ${ }^{* *} p<0.01$.
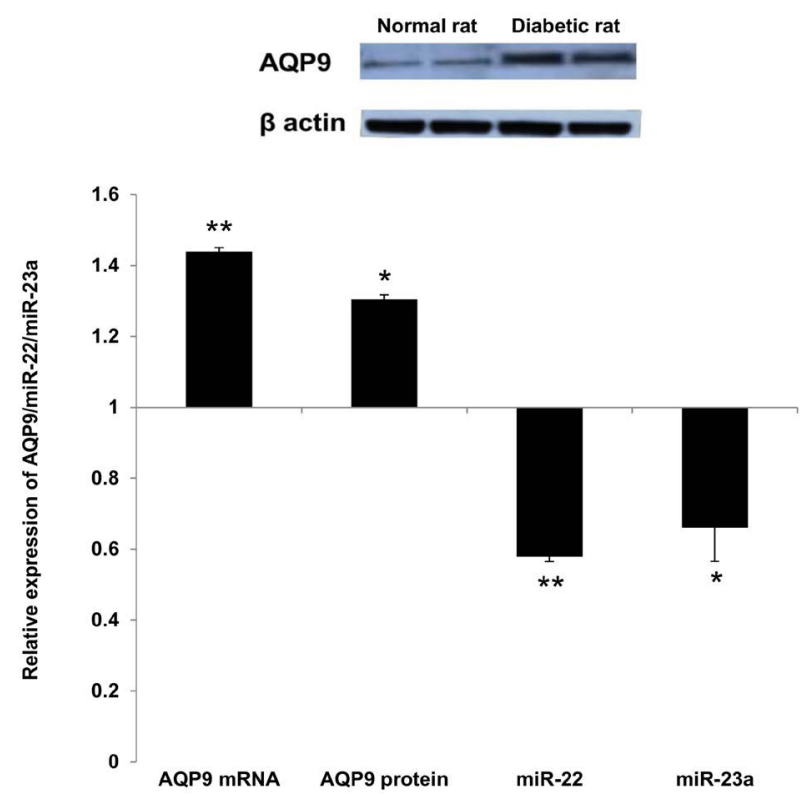

Figure 4: Altered expression of miR-22, miR-23a and $A Q P 9$ in the livers of diabetic rat models. In comparison to the corresponding controls, AQP9 was observed to be increased in liver samples of diabetic rats while both miR-22 and miR-23a showed significant down-regulation. Data was normalized against the housekeeping gene $18 \mathrm{~S}$ RNA and presented as mean \pm SEM (rats study $n=12$, 6 normal rats and 6 high fat diet+streptozotocin treated rats). AQP9 protein expression in the livers of normal and high fat diet+streptozotocin treated (Diabetic) rats is also shown (see insert). $\beta$-actin was used as loading control. The data presented here is a representative of six independent experiments

hours) post-transfection, the media was replaced with glucose and phenol free DMEM which does not contain the main gluconeogenic

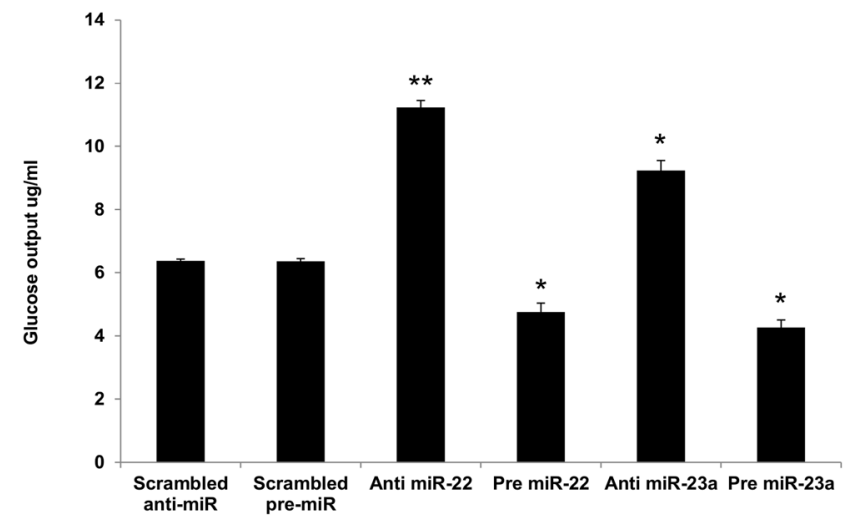

Figure 5: Relative glycerol-dependent glucose production in HepG2 cells HepG2 cells were transfected with anti or pre miR-22 or miR-23a (30 nM) for a period of 48 hour. Post-transfection cells were supplemented with $0.5 \mathrm{mM}$ glycerol as the primary gluconeogenic substrate and incubated for another 8 hours for glucose production to take place. At the end of 8 hours incubation, medium was collected in tubes for measurement of glucose productions (glucose assay). Medium collected from untreated cells without glycerol was used as blank. Data presented as mean $\pm \operatorname{SEM}(n=3)$ of glucose output in $\mu \mathrm{g} /$ $\mathrm{ml}$ against control cells transfected with corresponding anti or pre scrambled miRNAs. Statistically significant differences are tested at $p<0.05$ significance. ${ }^{*} p<0.05,{ }^{* *} p<0.01$

precursors such as lactate, glutamine and pyruvate. These cells were then supplemented with $0.5 \mathrm{mM}$ of glycerol as the primary gluconeogenic substrate [5]. Introduction of anti- miR-22 or miR-23a was found to increase glucose production by approximately 1.8 and 1.4 fold respectively (Figure 5). In contrast, transfection of pre- miR-22 and miR-23a could reduce glycerol gluconeogenesis by about -1.3 and -1.5 fold respectively. These results provided evidence that both miR-22 and miR-23a play important roles in the regulation of $A Q P 9$ as the glycerol importer and could therefore be as therapeutic targets in diabetes, particularly in the management of glycerogluconeogenesis.

\section{Discussion}

Impairment in gluconeogenesis is one of the many biological implications in diabetes and many have reported that gluconeogenesis is often elevated in T2D [17-19]. Among the many gluconeogenic substrates, glycerol is of particular interest because its availability as a gluconeogenesis precursor is increased in obese T2D individuals [20]. $A Q P 9$ has been identified as the primary route of hepatocyte glycerol uptake for glycerol gluconeogenesis and that its permeability can be inhibited by HTS13286 [5]. Despite having a great potential as an AQP9 inhibitor, HTS13286 exhibits limited solubility in $1 \%$ DMSOcontaining aqueous solution. miRNAs have long been associated to several pathological conditions including diabetes and several are currently being developed as therapeutic agents. In view of this, finding the direct miRNA-based regulator of $A Q P 9$ will definitely be of great advantage in the treatment of diabetes.

We have identified miR-22 and miR-23a as modulators of AQP9 expression (Figure 2). Over-expression of either miRNA was able to reduce $A Q P 9$ expression while inhibiting the miRNA expression resulted in increased $A Q P 9$ mRNA (Figure 3). These observations were also reflected at protein level with the exception of pre miR-23a treated cells that although there was reduced $A Q P 9$ protein expression, it was not statistically significant. This could possibly due to the moderate decrease of AQP9 mRNA in the pre miR-23a treated cells. A higher dose of pre miR-23a could be needed to bring down $A Q P 9$ protein to a significant level. Hepatic expression of $A Q P 9$ was found to be 
Citation: Karolina DS, Armugam A, Sepramaniam S, Pek SLT, Wong MTK, et al. (2014) miR-22 and miR-23a Control Glycerol-Dependent Gluconeogenesis by Regulating Aquaporin 9 Expression. Metabolomics S2: 002. doi:10.4172/2153-0769.S2-002

elevated in streptozotocin-treated mice [2,21]. Glucose production was also observed to be increased in parallel with the amount of glycerol perfused into the portal vein of insulin resistant $d b+/ d b+$ mice [2]. Likewise, we observed increased expression of $A Q P 9$ mRNA and protein in the livers of our diabetic rat model (Figure 4). These findings suggested that the dysregulated augmentation of $A Q P 9$ contributed to the increase in glycerol supply as a substrate for gluconeogenesis and therefore aggravating the hyperglycemic condition.

In the current study, we also demonstrated that down-regulation of $A Q P 9$ expression by increasing either miR-22 or miR-23a level resulted in significantly decreased glycerogluconeogenesis (Figure 5). Moreover, miR-23a has recently been reported to compromise gluconeogenesis by targeting G6PC which encodes the key gluconeogenic enzyme glucose-6phosphatase as well as the transcription factor $P g c-1 \alpha$ [8]. This indicates that not only miR-23a depletes the glycerol pool as a gluconeogenic substrate by inhibiting AQP9 upstream but it also represses G6PC and $P g c-1 \alpha$ and therefore exerting further suppression on gluconeogenesis. Although reduction of $A Q P 9$ protein was not found to be significant in the pre miR-23a treated cells, glycerogluconeogenesis in these cells exhibited significant decrease (Figure 5). In fact, the inhibitory effects of pre miR-23a on glycrogluconeogenesis was higher than miR-22. These data possible suggest that the lowering of glycerogluconeogenesis is more likely due to stronger suppression of miR-23a on G6PC and $P g c-1 \alpha$ rather than $A Q P 9$. Our study has highlighted miR-22 and miR$23 \mathrm{a}$ as promising lead compounds that could regulate $A Q P 9$ expression in the management of diabetes. Increasing the expression of miR-22 and miR-23a could suppress $A Q P 9$ expression and therefore control glycerogluconeogenesis, a process which is often increased in diabetes.

\section{Acknowledgements}

This work was supported by research grants, R-184-002-165-281 \& R-183000-290-213 from the National Research Foundation (CRP Program) and the National Medical Research Council, Singapore respectively.

\section{References}

1. Nurihan N, Consoli A, Gerich J (1992) Increased lipolysis and its consequences on gluconeogenesis in non-insulin-dependent diabetes mellitus. J Clin Invest 89: 169-175.

2. Kuriyama H, Shimomura I, Kishida K, Kondo H, Furuyama N, et al. (2002) Coordinated regulation of fat-specific and liver-specific glycerol channels, aquaporin adipose and aquaporin 9. Diabetes 51: 2915-2921.

3. Maeda N, Funahashi T, Shimomura I (2008) Metabolic impact of adipose and hepatic glycerol channels aquaporin 7 and aquaporin 9. Nat Clin Pract Endocrinol Metab 4: 627-634

4. Rojek AM, Skowronski MT, Füchtbauer EM, Füchtbauer AC, Fenton RA, et al. (2007) Defective glycerol metabolism in aquaporin 9 (AQP9) knockout mice. Proc Natl Acad Sci U S A 104: 3609-3614.

5. Jelen S, Wacker S, Aponte-Santamaría C, Skott M, Rojek A, et al. (2011) Aquaporin-9 protein is the primary route of hepatocyte glycerol uptake for glycerol gluconeogenesis in mice. J Biol Chem 286: 44319-44325

6. van Rooij E, Purcell AL, Levin AA (2012) Developing microRNA therapeutics. Circ Res 110: 496-507.

7. van Rooij E (2011) The art of microRNA research. Circ Res 108: 219-234.

8. Wang B, Hsu SH, Frankel W, Ghoshal K, Jacob ST (2012) Stat3-mediated

This article was originally published in a special issue, MicroRNAs and Metabolic Regulation handled by Editor(s). Dr. Kai Wang, Institute for Systems Biology, USA, Dr. David Galas, University of California, USA. activation of microRNA-23a suppresses gluconeogenesis in hepatocellular carcinoma by down-regulating glucose-6-phosphatase and peroxisome proliferator-activated receptor gamma, coactivator 1 alpha. Hepatology 56: 186-197.

9. Takagi S, Nakajima M, Kida K, Yamaura Y, Fukami T, et al. (2010) MicroRNAs regulate human hepatocyte nuclear factor 4 alpha, modulating the expression of metabolic enzymes and cell cycle. J Biol Chem 285: 4415-4422.

10. Gregersen LH, Jacobsen A, Frankel LB, Wen J, Krogh A, et al. (2012) MicroRNA-143 down-regulates Hexokinase 2 in colon cancer cells. BMC Cancer 12: 232

11. Karolina DS, Armugam A, Tavintharan S, Wong MT, Lim SC, et al. (2011) MicroRNA 144 impairs insulin signaling by inhibiting the expression of insulin receptor substrate 1 in type 2 diabetes mellitus. PLoS One 6: e22839.

12. Chutkow WA1, Patwari P, Yoshioka J, Lee RT (2008) Thioredoxin-interacting protein (Txnip) is a critical regulator of hepatic glucose production. J Biol Chem 283: $2397-2406$.

13. Wen YA, Liu D, Xiao YY, Luo D, Dong YF, et al. (2009) Enhanced glucose synthesis in three-dimensional hepatocyte collagen matrix. Toxicol In Vitro 23: 744-747.

14. Chan CB, Liu X, He K, Qi Q, Jung DY, et al. (2011) The association of phosphoinositide 3-kinase enhancer $A$ with hepatic insulin receptor enhances its kinase activity. EMBO Rep 12: 847-854.

15. Diaz G, Melis M, Tice A, Kleiner DE, Mishra L, et al. (2013) Identification of microRNAs specifically expressed in hepatitis $C$ virus-associated hepatocellular carcinoma. Int J Cancer 133: 816-824.

16. He XX, Chang Y, Meng FY, Wang MY, Xie QH, et al. (2012) MicroRNA-375 targets AEG-1 in hepatocellular carcinoma and suppresses liver cancer cell growth in vitro and in vivo. Oncogene 31: 3357-3369.

17. Basu R, Schwenk WF, Rizza RA (2004) Both fasting glucose production and disappearance are abnormal in people with "mild" and "severe" type 2 diabetes. Am J Physiol Endocrinol Metab 287: E55-62.

18. Boden G, Chen X, Stein TP (2001) Gluconeogenesis in moderately and severely hyperglycemic patients with type 2 diabetes mellitus. Am J Physiol Endocrinol Metab 280: E23-30.

19. Bogardus C, Lillioja S, Howard BV, Reaven G, Mott D (1984) Relationships between insulin secretion, insulin action, and fasting plasma glucose concentration in nondiabetic and noninsulin-dependent diabetic subjects. $\mathrm{J}$ Clin Invest 74: 1238-1246.

20. Yki-Järvinen H, Kubo K, Zawadzki J, Lillioja S, Young A, et al. (1987) Dissociation of in vitro sensitivities of glucose transport and antilipolysis to insulin in NIDDM. Am J Physiol 253: E300-304.

21. Carbrey JM, Gorelick-Feldman DA, Kozono D, Praetorius J, Nielsen S, et al. (2003) Aquaglyceroporin AQP9: solute permeation and metabolic control of expression in liver. Proc Natl Acad Sci U S A 100: 2945-2950. 